\title{
Ligand Release Pathways Obtained with WExplore: Residence Times and Mechanisms Supplemental Information
}

Alex Dickson, Samuel D. Lotz

(Dated: May 27, 2016)
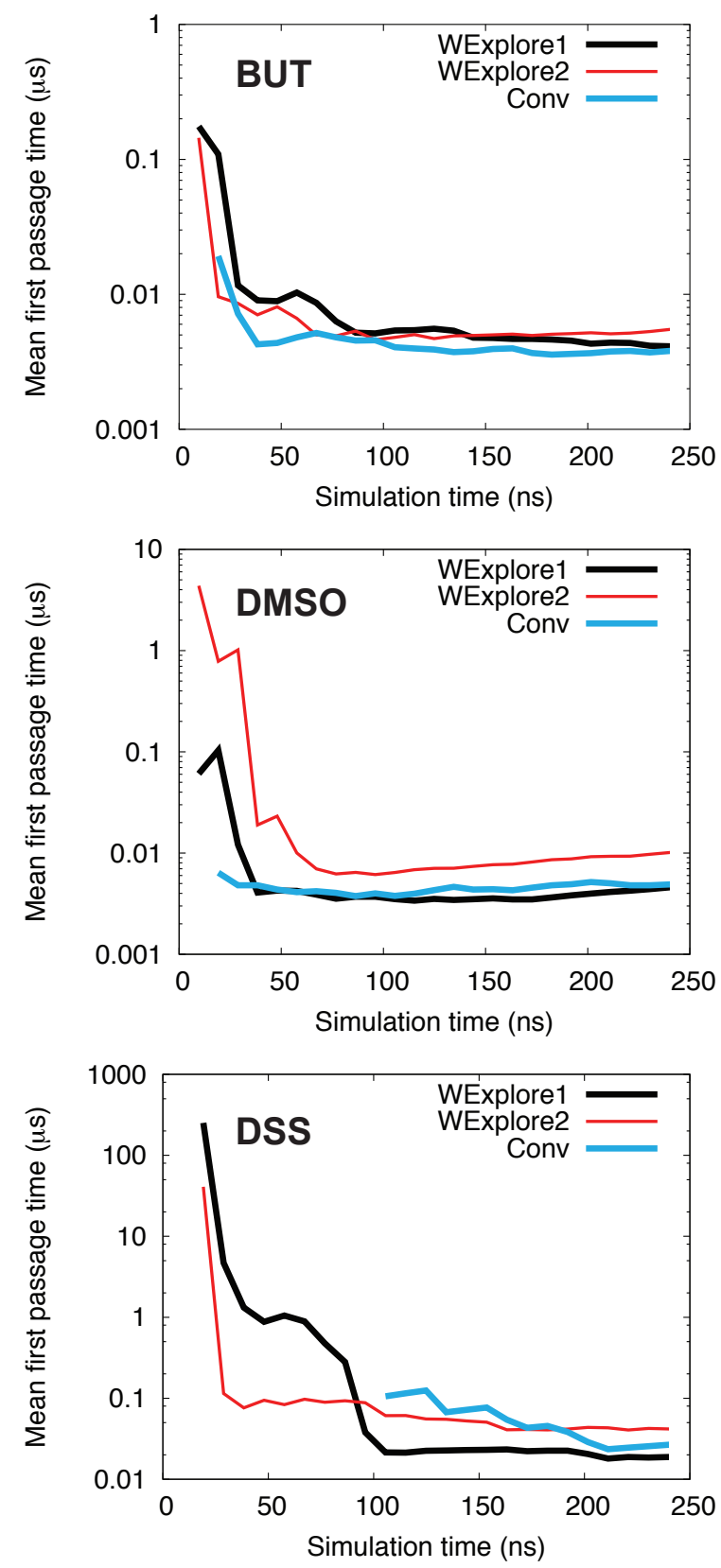

FIG. S1: Estimated mean first passage times for unbinding as a function of simulation time. Every 10 simulation cycles the best estimate of the unbinding mean first passage time (MFPT) is computed, and is shown here for both WExplore simulations and a conventional sampling simulation for each ligand. The exit rates are computed as described in the Methods section, and the MFPT is the inverse of this rate. 

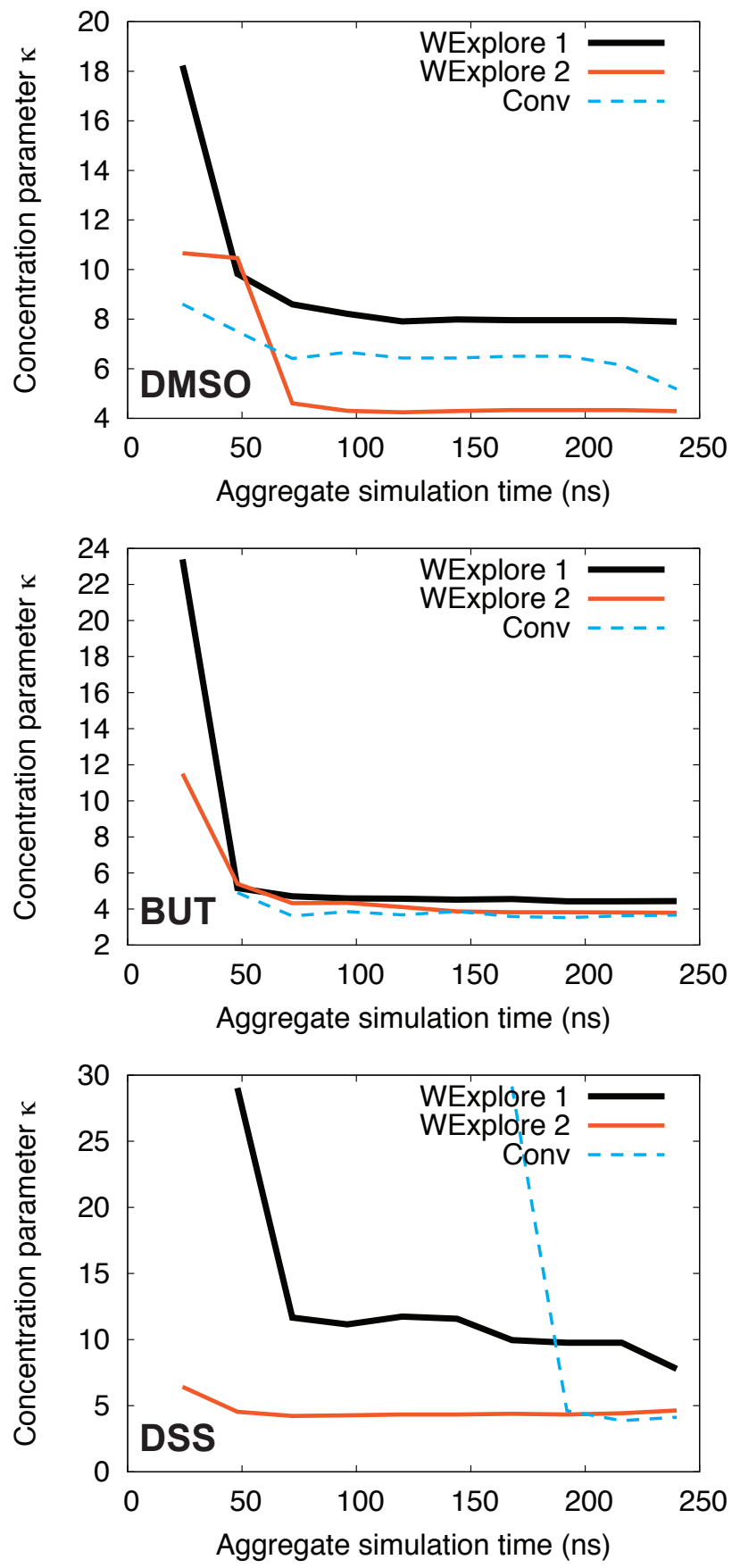

FIG. S2: Estimated concentration parameter values as a function of simulation time. The simulations are divided into 10 windows, and for each window with at least two exit points we estimate the concentration parameter $\kappa$ of the exit point distribution. Separate curves are shown for the two WExplore simulations and the single straight forward simulation conducted for each ligand. The final points in each curve have a standard deviation of 1.89, 0.41 and 1.98 units for DMSO, BUT and DSS, respectively. 


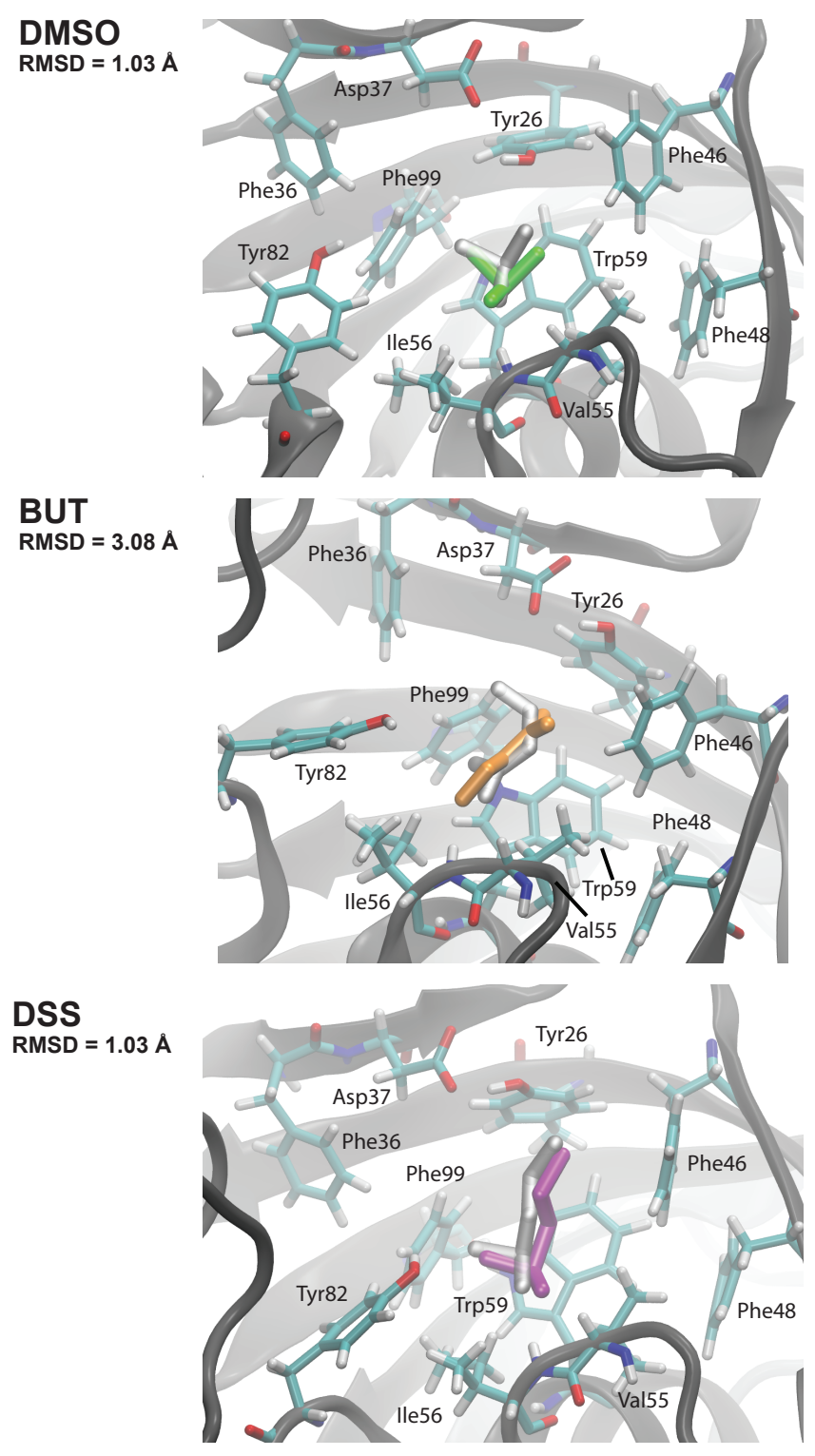

FIG. S3: Comparison of highest probability poses with crystallographic poses. Representative structures from the highest probability clusters are shown for each ligand. Residues that compose the binding pocket are shown in stick representation, and are labeled. The ligand pose from simulation is shown in color: green for DMSO, orange for BUT and purple for DSS. The corresponding crystallographic ligand pose, after alignment to the set of local protein residues shown here, is shown in white. The position of the ligand agrees very well between the two sets of structures, although the orientation of BUT is flipped. 